\title{
The Cosmic Ray Spectrum above 0.1 EeV measured by the Telescope Array and TALE Fluorescence Detectors
}

\author{
JiHee Kim*, Dmitri Ivanov, and Gordon Thomson for the Telescope Array \\ Collaboration \\ University of Utah, Department of Physics \& Astronomy and High Energy Astrophysics Institute, \\ Salt Lake City, Utah, USA \\ E-mail: jihee.kimecosmic.utah.edu
}

The Telescope Array (TA) is the largest cosmic ray detector in the Northern hemisphere. Initially designed to study ultra high energy cosmic rays of energies above $10^{18} \mathrm{eV}$. The Telescope Array Low-energy Extension (TALE) extends the sensitivity of TA down to $10^{15.6} \mathrm{eV}$. The original TA consists of three fluorescence detectors, viewing $3^{\circ}$ to $31^{\circ}$ in elevation each, and overlooking an array of scintillation counters. TALE adds additional fluorescence telescopes at the TA Middle Drum (MD) fluorescence detector site, and overlooking a graded infill array of counters. Together, TALE and MD see a combined sky coverage of $114^{\circ}$ in azimuth and 3-59 in elevation. In this work, we present a first measurement of the energy spectrum made by the combined TALE and MD fluorescence detectors operating in monocular mode.

35th International Cosmic Ray Conference - ICRC2017

10-20 July, 2017

Bexco, Busan, Korea

\footnotetext{
* Speaker.
} 


\section{Introduction}

The Telescope Array (TA) is deployed in Millard Country, Utah. It observes Ultra High Energy Cosmic Rays (UHECRs), using both fluorescence telescopes and scintillator surface detectors. A map of the detectors is shown in Figure 1. Using the fluorescence telescopes, we measure the scintillation light produced by air molecules during the development of cosmic ray induced air showers. Three telescope stations overlook the scintillator array. The Middle Drum (MD) station [1] is in the northern most location of TA and the Black Rock and Long Ridge fluorescence stations [2] are located in the southeast and the southwest, respectively. Additionally, an array of 507 scintillator surface detectors [3] were deployed in a square grid with $1.2 \mathrm{~km}$ spacing. The scintillator detector array is used to sample the density of shower particles arriving at the ground.

The Telescope Array has added an extension to lower the experiment's energy threshold allowing us to study cosmic rays with energies down below $10^{16} \mathrm{eV}$. This extension is known as the Telescope Array Low Energy extension (TALE) shown in Figure 2.

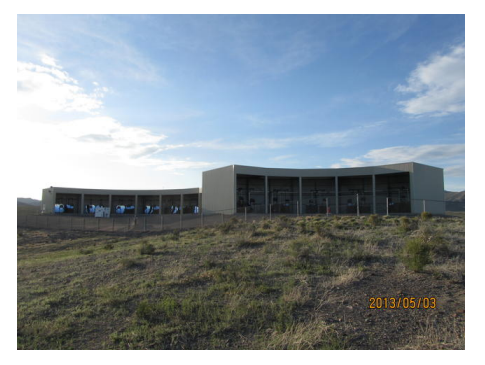

Figure 2: The lower building on the left is the main TA building contains 14 telescopes viewing $3-31^{\circ}$ elevation. The taller building on the right has 10 TALE telescopes viewing $31-59^{\circ}$ elevation above the main TA telescopes.

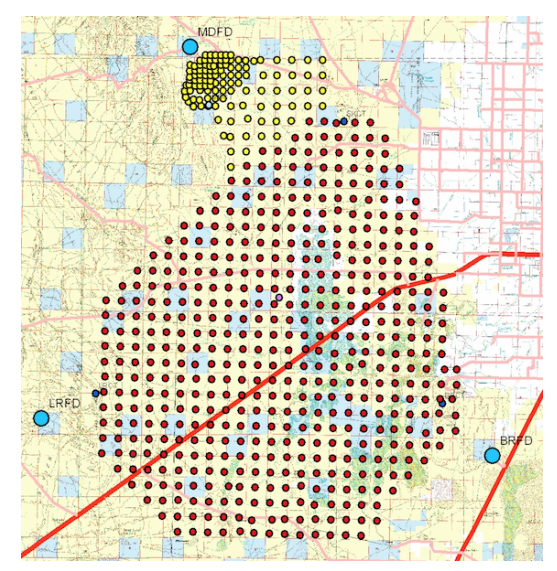

Figure 1: Map of Telescope Array detectors. Locations of the scintillator Surface Detectors (SD) are shown as red points and the locations of the three Fluorescence Detector (FD) stations are indicated by the blue points. Locations of the scintillator Surface Detectors for TALE are shown as yellow points. The purple point indicates the location of the Central Laser Facility (CLF).

High-elevation-angle telescopes and a graded infill array of more closely packed surface detectors were added to help make measurements of lower energy cosmic ray showers. While the Middle Drum (MD) telescopes view only $3^{\circ}-31^{\circ}$ in elevation, the TALE extension telescopes view the sky above the MD telescopes and increase the elevation viewing angle up to $59^{\circ}$. This enables us to observe the full development of lowerenergy showers which initiate higher in the atmosphere. With a larger field of view, we can see longer tracks and this gives us additional information about shower geometry and profile.

The goal of the MD and TALE spectral analysis is to determine the flux of cosmic rays and to produce a cross-calibrated monocular spectrum covering four decades in energy. For this analysis, 
we utilize a monocular reconstruction of the MD and TALE telescope data with events that triggered both Middle Drum and TALE fluorescence detectors. From this, we determine the geometries of the events observed and the energy of the primary particles.

\section{Event reconstruction and selection}

About 8 months of data from June 2014 to January 2015 were analyzed. The Middle Drum and TALE data and Monte Carlo events were processed using the same programs. By fitting tube viewing angles for the events to the arrival time of light at tubes from the shower, accurate geometrical reconstruction can be calculated. The Middle Drum and TALE data and Monte Carlo simulations are reconstructed in monocular mode with the geometry determined by the equation

$$
t_{i}=t_{0}+\frac{R_{p}}{c} \cdot \tan \left(\frac{\pi-\psi-\chi_{i}}{2}\right)
$$

where $t_{i}$ and $\chi_{i}$ are the trigger time and pointing direction of tube $i$, respectively; $\psi$ is the in-plane angle; $\mathbf{R}_{p}$ is the impact parameter of the shower; $c$ is the speed of the light; and $t_{0}$ is the time when the shower is calculated to be at $\mathrm{R}_{p}$. An example of the data event triggered by Middle Drum and TALE telescopes is shown in Figure 3.

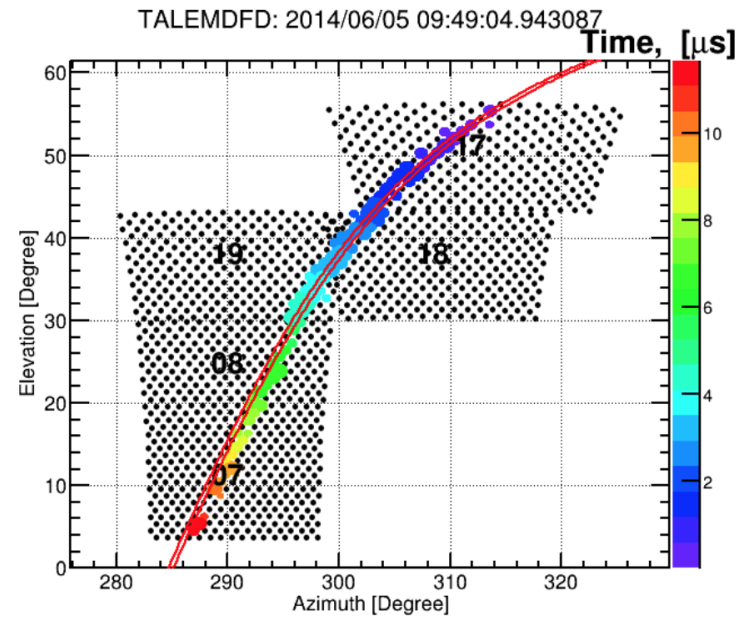

(a) Event display.

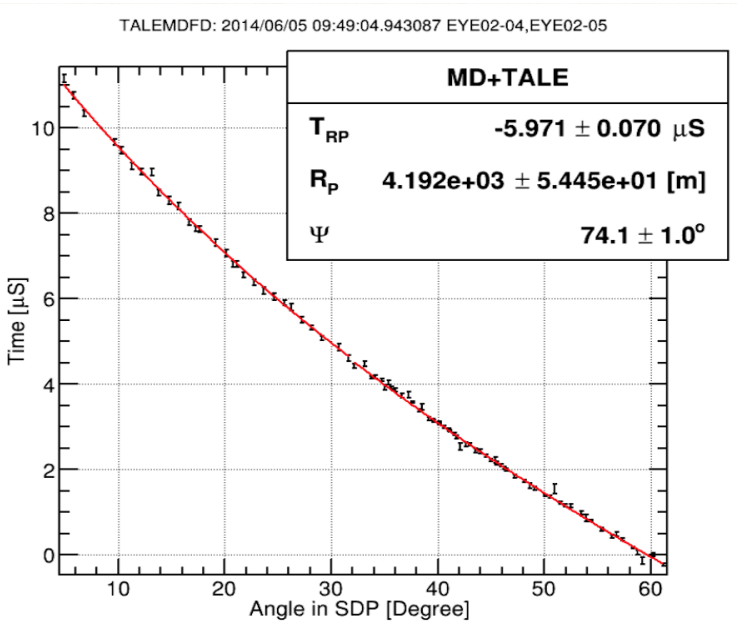

(b) Time versus Angle.

Figure 3: An example of the data event triggered by Middle Drum and TALE telescopes.

The profile of the shower is fitted using the Gaisser-Hillas parameterization formula [4]

$$
N_{e}(x)=N_{\max } \cdot\left[\frac{x-X_{0}}{X_{\max }-X_{0}}\right]^{\frac{X_{\max }-X_{0}}{\lambda}} \cdot e^{\frac{X_{\max }-x}{\lambda}},
$$

where $N_{e}(x)$ is the number of charged particles at a given slant depth, $x$, in $\mathrm{g} / \mathrm{cm}^{2} ; X_{\max }$ is the slant depth where the number of secondary particles reaches the maximum; $N_{\max }$ is the maximum number of particles at $X_{\max } ; X_{0}$ is a fit parameter associated with the depth of the first interaction; and $\lambda$ is a fit parameter explaining the width of the shower profile. 
In addition, a parameterization of mean energy deposit [5] was applied:

$$
\alpha_{\text {eff }}(s)=N \cdot \frac{c_{1}}{\left(c_{2}+s\right)^{c_{3}}}+c_{4}+c_{5} \cdot s,
$$

where $c_{i}$ are the constants from Nerling et al. [5]. The mean ionization loss rate, $\alpha_{\text {eff }}$, at a given shower age, $\mathrm{s}$, is determined in the condition at $\mathrm{E}_{c u t}=1 \mathrm{MeV}$. However, $\mathrm{E}_{\text {cut }}$ value in our program is $50 \mathrm{keV}$, so $\mathrm{N}$ indicates a scale normalization constant to correct for the difference in $\mathrm{E}_{\text {cut }}$.

To obtain good resolution and Data/Monte Carlo comparisons, quality cuts were performed on the fully reconstructed showers. These cuts were optimized for the fluorescence events observed by Middle Drum and TALE stations. An event is retained if:

- An event is successfully reconstructed. Additionally, $X_{\max }$ should be between the first and last profile bins.

- A clear peak in the profile should be observed and be bright enough. The the slant depth of the first triggered tube must fall greater than $200 \mathrm{~g} / \mathrm{cm}^{2}$ and profile reduced $\chi^{2}<10$.

- Geometry: the in-plane angle, $\psi$, is $<120^{\circ}$, to improve resolution. The duration of the event must be $>0.5 \mu \mathrm{sec}$ and the tracklength must be $>20^{\circ}$.

\section{Monte Carlo simulation}

CORSIKA simulated shower library with QGSJETII-03 for pure proton and pure iron primaries was used. These showers were thrown from $10^{16}$ to $10^{18} \mathrm{eV}$ with the spectral index of 3 . The Monte Carlo simulated an isotropic distribution in the azimuthal angle, $\mathrm{R}_{p}$ was between $10 \mathrm{~m}$ to $50 \mathrm{~km}$, and $\theta$ was thrown up to $80^{\circ}$. To reflect energy spectral features to our energy distribution, the most recent Telescope Array combined energy spectrum [6] was implemented by weighting on showers. In addition, HiResMIA mixed composition was employed by consulting with HiResMIA and HiResStereo data in order to derive proton fraction as a function of energies. Figure 4 and 5 show the resolution of Middle Drum and TALE monocular spectral analysis.
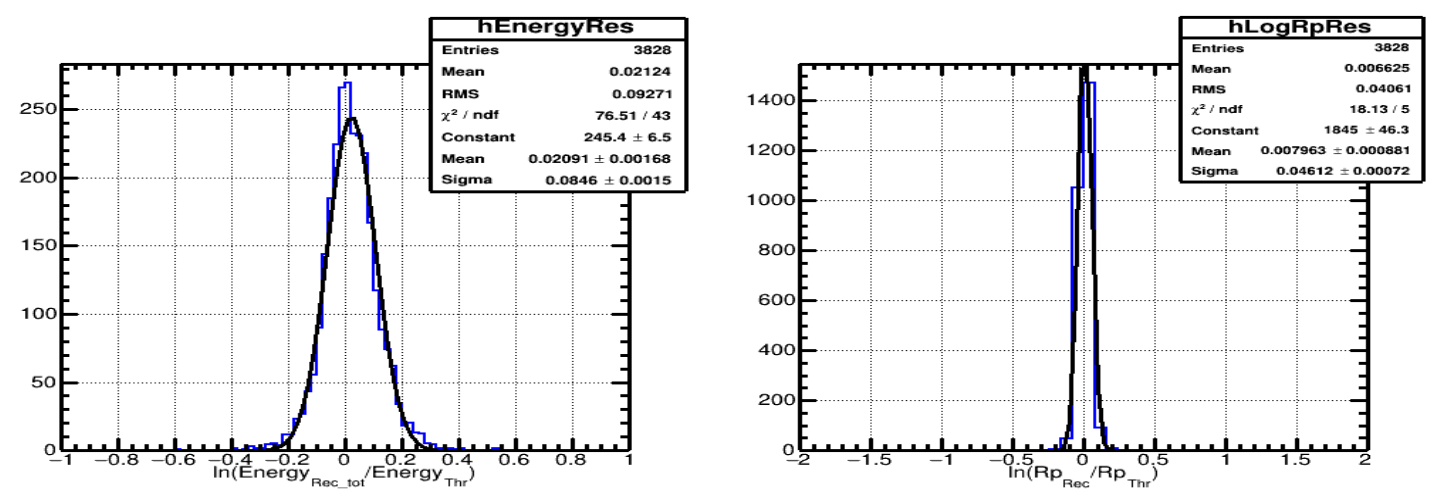

Figure 4: Resolution studies using Monte Carlo events. Left: the total energy of Monte Carlo simulated events shown. Right: the impact parameter, $\mathrm{R}_{p}$. The Gaussian fit is used to determine the detector bias and resolution. 

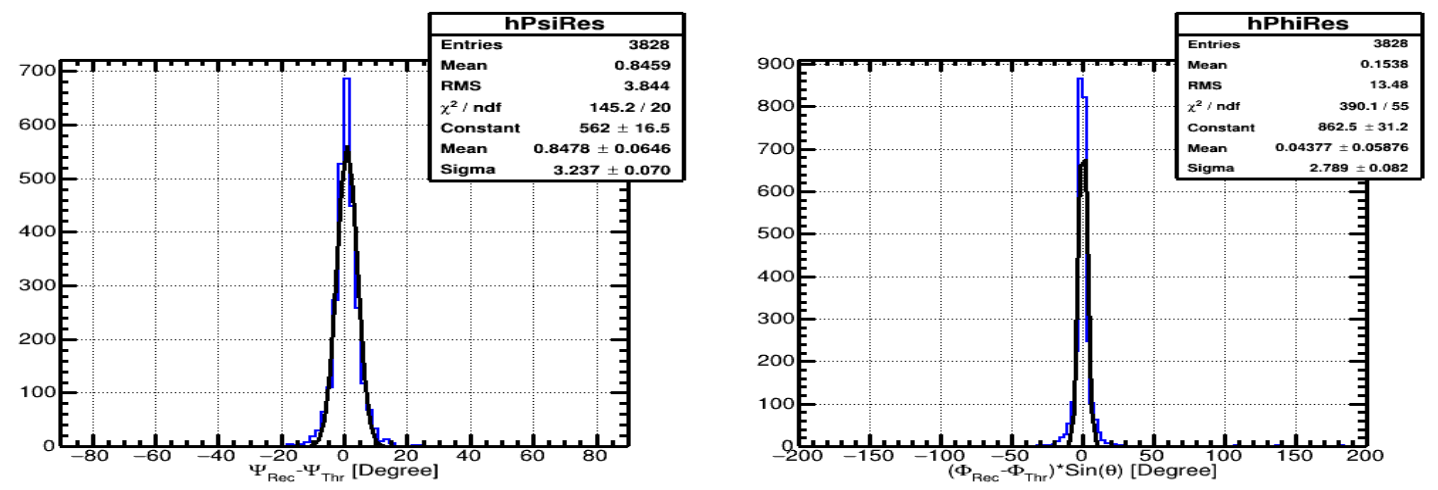

Figure 5: Resolution studies using Monte Carlo events. Left: the in-plane angle, $\psi$. Right: the azimuthal angle, $\phi$. The Gaussian fit is used to determine the detector bias and resolution.

\section{Data/MC comparisons}

To check if the Monte Carlo represents the data, measurable parameters' distributions were plotted. Among measurable parameters, the impact parameter and zenith angle parameter are important to determine the aperture. Figure 6 shows Data and Monte Carlo comparisons for energy, impact parameter, zenith angle, and azimuthal angle. Black data points and red Monte Carlo histogram are in good agreement.
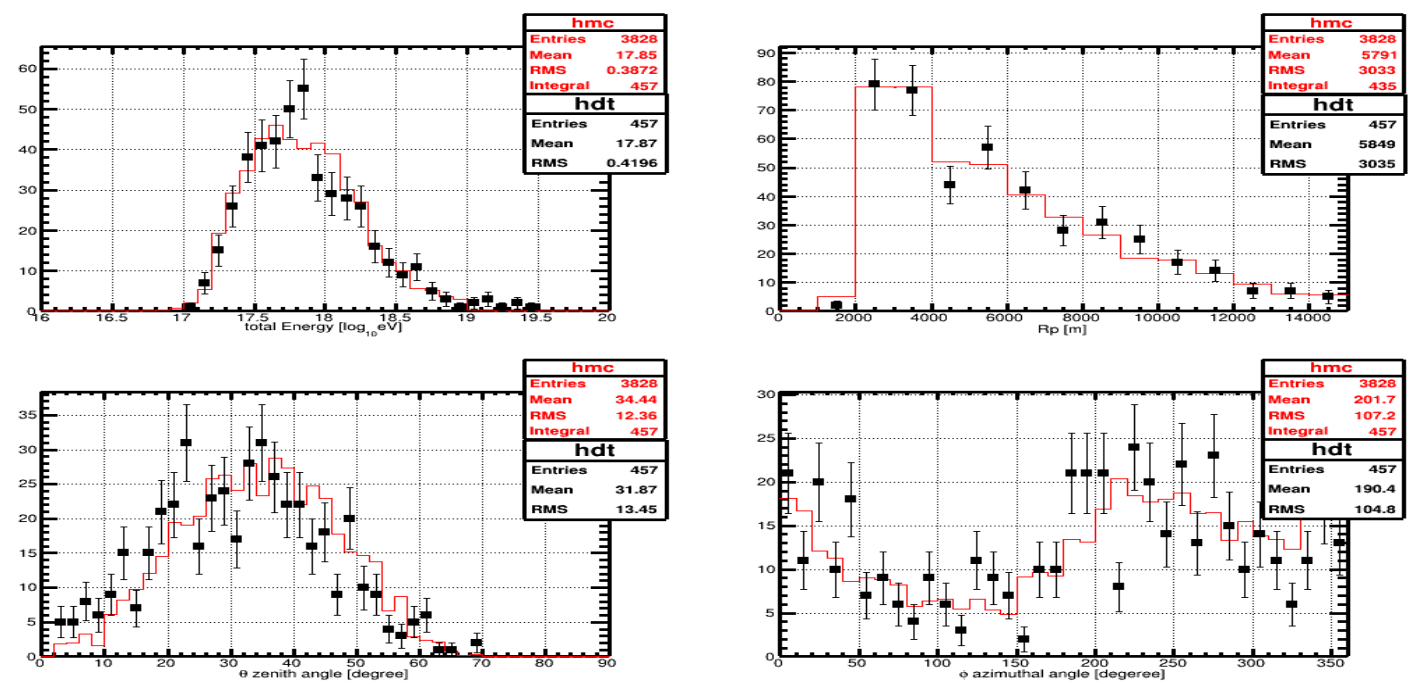

Figure 6: Data/Monte Carlo comparisons. Top left: the total energy. Top right: the impact parameter, $\mathrm{R}_{p}$. Bottom left: the zenith angle, $\theta$. Bottom right: the azimuthal angle, $\phi$. The black points with error bars show the data, while the Monte Carlo is shown by the red histogram. The Monte Carlo has been normalized to the same number of events as the data.

\section{Summary}

The Telescope Array has been extended to lower the experiment's energy threshold, allowing 
us to study cosmic rays with energies down below $10^{16} \mathrm{eV}$, which is called the Telescope Array Low Energy extension (TALE). The Telescope Array and TALE together enable us to observe the full development of lower-energy showers which initiate higher in the atmosphere and observe cosmic rays covering four decades in energy using the same suite of detectors. For this analysis, we use a monocular reconstruction of the MD and TALE telescope data with events that triggered both Middle Drum and TALE fluorescence detectors. TA combined energy spectrum presented at ICRC2015 and HiResMIA composition were assumed. The resolution of the shower energy and geometric variables shows good agreement between the thrown and reconstructed values. Data and Monte Carlo comparisons for energy and geometrical variables are in good agreement.

\section{Acknowledgement}

The Telescope Array experiment is supported by the Japan Society for the Promotion of Science through Grants-in-Aid for Scientific Research on Specially Promoted Research (21000002) "Extreme Phenomena in the Universe Explored by Highest Energy Cosmic Rays" and for Scientific Research (19104006), and the Inter-University Research Program of the Institute for Cosmic Ray Research; by the U.S. National Science Foundation awards PHY-0601915, PHY-1404495, PHY1404502, and PHY-1607727; by the National Research Foundation of Korea (2015R1A2A1A01006870, 2015R1A2A1A15055344, 2016R1A5A1013277, 2007-0093860, 2016R1A2B4014967); by the Russian Academy of Sciences, RFBR grant 16-0200962a (INR), IISN project No. 4.4502.13, and Belgian Science Policy under IUAP VII/37 (ULB). The foundations of Dr. Ezekiel R. and Edna Wattis Dumke, Willard L. Eccles, and George S. and Dolores Doré Eccles all helped with generous donations. The State of Utah supported the project through its Economic Development Board, and the University of Utah through the Office of the Vice President for Research. The experimental site became available through the cooperation of the Utah School and Institutional Trust Lands Administration (SITLA), U.S. Bureau of Land Management (BLM), and the U.S. Air Force. We appreciate the assistance of the State of Utah and Fillmore offices of the BLM in crafting the Plan of Development for the site. Patrick Shea assisted the collaboration with valuable advice on a variety of topics. The people and the officials of Millard County, Utah have been a source of steadfast and warm support for our work which we greatly appreciate. We are indebted to the Millard County Road Department for their efforts to maintain and clear the roads which get us to our sites. We gratefully acknowledge the contribution from the technical staffs of our home institutions. An allocation of computer time from the Center for High Performance Computing at the University of Utah is gratefully acknowledged.

\section{References}

[1] D.C. Rodriguez et al. for the Telescope Array Collaboration, Astroparticle Physics 39-40 (2012) 109-119

[2] Y. Tameda et al. for the Telescope Array Collaboration, Nuclear Instruments and Methods in Physics Research A 609 (2009) 227-234

[3] T. Nonaka et al. for the Telescope Array Collaboration, Nuclear Instruments and Methods in Physics Research A 689 (2012) 87-97 
[4] T. K. Gaisser and A. M. Hillas, "Reliability of the method of constant intensity cuts for reconstructing the average development of vertical showers", in Proceedings of the International Cosmic Ray Conference, vol. 8 (1977) 353-357

[5] F. Nerling, J. Blumer, R. Engel, M. Risse, Astroparticle Physics 24 (2006) 421-437

[6] D. Ivanov et al. for the Telescope Array Collaboration (2015) "TA Spectrum Summary", PoS ICRC2015 (2016) 349. 